\title{
Public health strategies to combat opioid crisis in the United States
}

\author{
Festina Balidemaj \\ Lunds Universitet Medicinska Fakulteten, Lund, Sweden \\ Northwestern Health Sciences University, Minneapolis, Minnesota, USA
}

\section{Abstract}

Background: The opioid epidemic in the United States is a national public health crisis. Driven by an increase in availability of pharmaceutical opioids and by an increase in their consumption, specifically, for pain treatment, more so in the past twenty years, it has led to an economic cost of prescription opioid abuse, overdose, and dependence in the United States estimated to be 78.5 billion USD. Methods: A thorough evaluation of the relevant extracted literature has been used to answer the question of the most effective ways to regulate health markets to decrease the opioid crisis in the United States, using keywords and phrases such as opioid epidemic in the US, prescription drug abuse, prescription medication abuse in US, medication misuse, costeffective ways to health market regulation, drug monitoring programs, prescriber continuing education, provider continuing education, and pain management optimization. Results: Overcoming this epidemic nationwide requires improvement in patient utilization of and access to safe and effective treatment options for opioid abuse and overdose, addressing the stigma correlated with opioid use, considering appropriate use of abuse deterrent formulations (ADF) along with patient education, and improving prescribing practices via utilization of drug monitoring programs, CDC opioid prescribing guidelines and provider continuing education. Conclusion: Utilizing and implementing the aforementioned steps has shown to be a challenge. Further and repeated attempts are needed, while at the same time considering possible new steps that could help reinforce their utilization further.

Keywords: Opioid Crisis, Public Health, Public Health Strategies, Opioid Epidemic in the US

\section{Introduction}

One of the most common reasons that patients go to seek medical care in United States is pain. And every year, approximately 100 million people in the US suffer from pain, of which $9-12 \%$ report the pain to be chronic. As a result, in 2016, about 214 million prescriptions for opioid medications (the most commonly prescribed 
medications in the US [6]) were written and dispensed. In turn, this has risen, in parallel, a phenomenon known as the opioid crisis, including opioid overdose deaths and opioid addictions [3,4\&5]. While in today's modern medical practice there are numerous ways to treat pain, one way includes use of opiates, which have officially been approved for analgesia (pain relief) for almost 70 years. While this class of drugs has been in use for a very long time, it is in the past two decades that a concern about their safety was raised by many reports. While cases of opiate overdose and toxicity all over the United States are continuously reported, the number of prescriptions for opiates has also significantly increased over the past 20 years [1]. According to the Centers for Disease Control and Prevention, as well as the Drug Enforcement Agency of the US, the opiate diversion rate, number of prescriptions for opiates, and opiaterelated deaths have increased exponentially in the past two decades in the US. Pain management experts believe this high incidence of opiate overdose cases to be due to patients trying to manage unrelenting pain and not due to intentional causes [7]. Opioid overdose is a phenomenon that causes a person to have excessive unopposed stimulation of the opiate pathway. This can, in turn, cause respiratory depression and likely, death [1].

Currently in the US, overdose from drugs is a leading cause of accidental death, opioids being the leading drug class. Approximately one thousand emergency department visits everyday are due to opioid misuse. In addition, opioid overdose is responsible for an average of ninety-one US deaths per day [7]. This nationwide public health crisis is continuing to escalate. The national rate of opioid-related hospitalizations between 2005 and 2014 increased 64\%, reaching 225 hospitalizations per population of 100,000 . There has also been a $27 \%$ increase in death rate due to opioid overdose or toxicity from 2015 to 2016, with 42,000 Americans dying from this cause. This epidemic is widespread throughout the United States, while sociodemographic and regional variations exist [2].

While the rate of prescribing opioids in the recent years has declined, the increased use and availability of pain opioid medications have played a crucial role in this modern-day epidemic. The amount of opioid prescriptions in the US was triple in 2016 when compared to its amount in 1999. As a result, more than 17,000 people died from an overdose of pharmaceutical opioids. It's been found that even proper prescribing and use of pharmaceutical opioids leads to adverse events, such as addiction and fatal toxicity or overdoses, showing a statistically spatial and temporal relationship between pharmaceutical opioid availability and mortality due to overdose [2]. The opioid epidemic has been referred to as the 'most consequential preventable public health problem in the US' pointing out that taking appropriate preventable measures, the issue has a chance of improving immensely [10].

Economic cost of prescription opioid abuse, overdose, and dependence from a societal perspective in the US is estimated to be 78.5 billion USD, of which a third of it is due to increased substance-abuse treatment costs and increased healthcare costs, 
approximately adding up to 28.9 billion USD. In addition, about $25 \%$ of the total burden is borne by the public sector in healthcare, criminal justice costs, lost productivity (such as premature death due to opioid dependence or abuse, incarceration, and loss of productive hours [household productivity or paid employment] due to dependence or abuse), and substance abuse treatment costs [15].

A crucial component in identifying preventive cost-effective strategies is to first understand the economic burden that is produced by adverse health events due to the opioid crisis. The most recent estimate of the overall societal impact of prescription opioid dependence, misuse, and abuse in the US was found to be substantially higher than it was a decade ago, 55.7 billion USD [15]. And while the epidemic has continued to progress throughout the past two decades, understanding the economic burden caused by the adverse health outcomes due to the opioid epidemic is key to identifying appropriate preventive strategies that are cost effective.

Opioid abuse and toxicity epidemic are not solely an American occurrence and concern, it is a global problem. It is estimated that $0.4 \%$ of the population (close to 20 million people) regularly use derivatives of opioids, such as heroin or opium [2]. Having a better understanding of effective ways to regulate the market can lead to decreased incidence and prevalence of the opioid crisis not only in the US but internationally as well.

\section{Methods}

A thorough literature review has been conducted using trusted sites, such as PubMed, Lund University online library, Clemson University online library and Northwestern Health Sciences University online library. Keywords used included opioid epidemic in the US, prescription drug abuse, prescription medication abuse in US, medication misuse, cost-effective ways to health market regulation, drug monitoring programs, prescriber continuing education, provider continuing education, pain management optimization, etcetera. A thorough evaluation of the relevant extracted literature has been used to answer the question of the most effective ways to regulate health markets to decrease the opioid crisis in the United States.

\section{Results}

In order to contribute towards decreasing the incidence and prevalence of opioid abuse and toxicity, both factors leading to the current opioid crisis in the United States, there are several steps that can be addressed and improved upon at this time. First, improvement is needed in patient utilization of and access to safe and effective treatment options for opioid overdose. Second, stigma correlated with opioid use must be addressed. Third, abuse deterrent formulations (ADF) need to be considered in appropriate cases along with patient education. Lastly, prescribing practices must be improved via utilization of drug monitoring programs and provider continuing education. 


\section{Improving utilization of and access to safe and effective treatment options}

Three main FDA-approved medications are available in the market to treat opioid use disorder. Those include buprenorphine, methadone, and naltrexone. Buprenorphine and methadone are long-acting receptor agonists, providing consistent drug levels systemically in blood and reduce opioid cravings and reduce withdrawal symptoms. According to Lyden et al. numerous studies have shown that buprenorphine and methadone reduce illicit use of opioids, increasing retention of patients in drug treatment plans, and reduce mortality. However, despite the positive evidence, these opioid agonists remain underutilized for multiple reasons, including lack of prescriber and provider training to initiate use of medications and consultations with patients to maintain treatment when needed, misconceptions by healthcare providers and patients about how these treatment drugs work and their outcomes, and worry of criminalization or stigma by the patients. Naltrexone is an opioid receptor antagonist and blocks the effects of opioids, making it impossible for the patient to enjoy the euphoric effects of opioids and, in turn, reducing relapse [2]. In addition, naloxone, which is also a pure competitive antagonist at the opiate receptors is FDA approved to combat opioid overdose. While it can be administered intravenously and nasally by a healthcare provider, recently a hand-held autoinjector has been approved, where caregivers or family members are able to administer the drug and intervene with an overdose of opioid [1].

\section{Addressing the stigma correlated with opioid use}

Addressing the stigma that is correlated to opioid use is necessary as it has been found that when restrictive policies are enforced and people breaking said policies are categorized and stigmatized as criminals, drives people to engage in risky behavior, in this case inject in risky conditions, leading to HIV and hepatitis C incidences due to needle sharing. Hence, prohibition of opioid use leads to infectious diseases. Removing the stigma associated with drug use and approaching policy reform through defining drug use as primarily a social and health matter, rather than a criminal matter will allow for a safer and a more effective approach to decreasing the opioid crisis [9]. In addition, stigmatizing drug use will prevent patients willing to get treatment from asking for professional help due to fear of criminalization [2].

In order to address the stigma correlated with drug use, many studies have found that the language used in discussing and treating patients who are dealing with substance abuse or substance use disorders is among the most important factors. Appropriate language, which will be explained, will help remove treatment barriers for patients and remove stereotypes. For example, avoiding use of terms such as 'substance abuser' or 'addict' will help avoid putting the patient at fault of their situation. In addition, using 'person first' language has been found to be successful in addressing stereotypes and stigma in opioid use disorders by multiple studies. In 'first person' language, description of the individual precedes the description of their disorder or 
diagnosis. For example, use 'woman/man with history of opioid use' instead of 'opioid abuser' [2].

\section{Abuse deterrent formulations (ADF) and patient education}

ADF medications are opioid analgesics available with prescription only. They are technologically designed to make them more difficult for the patient to abuse. Their chemical and physical properties resist any attempted manipulation or cause loss of the drugs' psychotropic effects. Post-marketing studies of ADFs have shown that ADF use leads to a less likely result of 'drug-liking' and 'likelihood to take drug again' by the patient, when compared to non-ADF opioid users. Kumar et al. found that in a 5year study, abuse-related costs using ADF opioids were substantially lower than nonADF opioid related costs, by 274 million USD. In addition, ADF opioids on average prevent 2300 new abuse cases and about 6600 abuse years when compared to nonADF opioids [3]. However, ADF opioids are overall more expensive that non-ADF opioids, hence, this option should be considered in a population that is at risk to try therapy manipulation or drug abuse, such as patients with history of drug diversion or abuse, patients with mental health diagnosis or problems, and possibly patients in long-term pain management. According to Kumar et al. it costs the health system approximately 232,000 USD in order to prevent a new opioid abuse case and about 1.4 billion USD in order to prevent one overdose by opioid death when using ADF opioid as opposed to non-ADF opioid [3].

In addition, continuous patient education by healthcare providers on the effects of opioid misuse and abuse should be practiced in order to ensure patients are aware of the seriousness of opioid use and the importance of taking these medications exactly as prescribed due to their highly addictive properties, as well as danger of accidental overdosing as well as drug-drug interactions.

\section{Improving prescribing practices via provider continuing education, CDC opioid prescribing guidelines, and drug monitoring programs}

As mentioned earlier, lack of utilization of treatment options, such as methadone or buprenorphine, which show high success rates, is due to lack of provider awareness to initiate and maintain such treatment in qualifying patients. In order to combat this issue, continuous provider continuing education is crucial. Prescribing opioids to treat pain will help patients in the short term, however long term-effects can be detrimental to the patient. In addition, in cases where a provider may know that a patient is highly likely to abuse opioids, he or she may choose to prescribe another alternative, Tramadol. Tramadol (brand-named Ultram) is a classified analgesic of a non-opiate class. It has a length of duration of action of 5-6 hours and it is safer than opiates when treating pain [1].

In addition, in order to address the serious detrimental adverse health outcomes (such as addiction and fatal overdoses) of opioid use even when prescribed by physicians and taken as directed, the CDC created specific guidelines in 2016 in order 
to assist clinicians to recognize patients with high-risk of opioid abuse and to properly prescribe opioids for treatment of pain. The 12 specific recommendations can be seen on Table 1 of Appendix A. Utilization of these guidelines needs to be followed in all states in order to avoid overprescribing opioids, especially in acute cases or brief emergency department visits.

Lastly, prescription drug monitoring programs must be utilized nationwide. While these programs have been established in many states in the US, not all are utilizing them, and no interstate data sharing is currently being done. Thus far, their use has been shown to improve prescriber confidence, identify patients that have multiple providers, and reduce overall availability of controlled substances $[2,12,13]$. Where implemented, these programs decreased opioid-related deaths by 1.12 per 100,000 population [16].

\section{Discussion}

Overcoming the opioid epidemic nationwide is a primary public health issue and utilizing the aforementioned factors and services will help in addressing many of the issues that are currently contributing towards the opioid crisis.

Decision makers at the state and federal levels have already responded to the opioid epidemic with multiple attempted strategies, all of which aimed to decrease its burden. For example, in 2011 the National Drug Control Policy had already called for all of the states to provide functional prescription drug monitoring programs and also encouraged the administrations to share the information and data within the monitoring programs both interstate and intrastate in order to catch multiple drug prescribing. However, attempts like these, often face challenges, such as financial constraints that need strategies that are both clinically effective as well as cost effective. In this case, the challenge to some providers seemed to be balancing care for patient that needed pain treatments and addressing the epidemic of opioid overdose at the same time [8,15]. In addition, some providers and dispensers have reported concerns in prescription drug monitoring programs difficulty of use, effectiveness and necessity of their advancement, suggesting that while these programs are effective, they need to be improved in order to increase the likelihood that providers will feel more comfortable using them [11,14].

In order to reinforce the utilization of steps that studies have shown to be successful in reducing the opioid crisis in the US but that are not being utilized sufficiently, such as use of prescription monitoring programs, use of available treatment options for opioid abuse, referral to the CDC opioid prescribing guidelines, it has been suggested that stricter rules and certain reinforcements and requirements may be helpful, such as, for example, making the use of prescription monitoring programs a requirement for physicians in order to receive their state prescriber license. In addition, states can educate physicians about the dangers of the opioids and the importance of identifying patients at risk for opioid abuse or misuse, as well as prescribing the appropriate available treatment options that are not currently being maximally used. States can 
even litigate against pharmaceutical manufacturers, as they had done while fighting tobacco companies and can even ask pharmaceutical companies to stop manufacturing a certain drug altogether [8], should they decide that its manufacturing is contributing to a public health issue that is unmanageable otherwise.

\section{Conclusion}

While attempts to combat the opioid epidemic have been made, the state and federal governments have only recently started to understand the magnitude of the seriousness of this public health crisis [8]. The methods with promising improvement of the situation have been identified and include utilization of and access to safe and effective treatment options for opioid abuse and overdose, addressing the stigma correlated with opioid use, considering appropriate use of abuse deterrent formulations (ADF) along with patient education, and improving prescribing practices via utilization of drug monitoring programs, CDC opioid prescribing guidelines and provider continuing education. However, utilizing and implementing these steps has shown to be a challenge. Further and repeated attempts are needed, while at the same time considering possible new steps that could help reinforce their utilization further.

\section{Funding}

No funding was received for the implementation of this study.

Conflict of Inerest

There is no conflict of interest from the author.

\section{Key Points}

- Attempts to combat the opioid epidemic have been made, and the state and federal governments have only recently started to understand the magnitude of the seriousness of this public health crisis.

- The methods with promising improvement of the situation have been identified.

- Utilizing and implementing the existing public health strategies has shown to be a challenge.

- Further and repeated attempts are needed, while at the same time considering possible new steps that could help reinforce their utilization further.

\section{References}

[1] Ali M, Henke R, Mutter R, O'Brien P, Cutler E, Mazer-Amirshahi M, Pines J. Family member opioid prescriptions and opioid use disorder. Addictive Behaviors. 2019; 95: 58-63. 
[2] Carnes N, Wright E, Norwood C. A qualitative analysis of prescribers' and dispensers' views on improving prescription drug monitoring programs. Social and Administrative Pharmacy. 2017; 13: 1167-1174.

[3] Center for Disease Control and Prevention. [Internet]. 2016 [cited 2019 Apr 23]; Available from: www.cdc.gov/drugoverdose/index.html

[4] Edmund Rose M. Are prescription opioids driving the opioid crisis? Assumptions vs facts. Pain Med. 2018 Apr; 19(4): 793-807.

[5] Florence C, Luo F, Xu L, Zhou C. The economic burden of prescription opioid overdose, abuse, dependence in the United States, 2013. Med Care. 2016; 54(10): 901-906.

[6] Gilson A, Fishman S, Wilsey B, Casamalhuapa C, Baxi H. Time series analysis of California's prescription monitoring program: Impact on prescribing and multiple provider episodes. American Pain Society. 2012; 13(2): 103-111.

[7] Kaye A, Atluri S, Sanapati J, Benyamin R, Hirsch J, Manchikanti L. Reframing the prevention strategies of the opioid crisis: Focusing on prescription opioids, fentanyl and heroin epidemic. 2018; 21: 309-326.

[8] Kazatchkine M, Bém P, Dreifus R. Why doctors should support regulated markets in illicit drugs. BMJ. [Internet]. 2018 Sep. [cited 2019 Apr 23]; Available from: blogs.bmj.com/bmj/2018/09/24/doctors-supportregulated-markets-illicit-drugs/

[9] Kumar V, Agboola F, Synnott P, Segel C, Webb M, Ollendorf D, Banken R, Chapman R. Impact of abuse deterrent formulations of opioids in patients with chronic pain in the United States: A cost-effectiveness model. Value in Health. 2019: 416-422

[10] Lyden J, Binswanger I. The United States opioid epidemic. Elsevier. 2019.

[11] Pardo B. Do more robust prescription drug monitoring programs reduce prescription opioid overdose? Addiction. 2016; 1773-1786.

[12] Patrick S, Fry C, Jones T, Buntin M. Implementation of prescription drug monitoring programs associated with reduction in opioid-related death rates. Health Affairs. 2016;

[13] Rutkow L, Turner L, Lucas E, Hwang C, Alexander G. Most primary care physicians are aware of prescription drug monitoring programs, but many find the data difficult to access. Health Affairs. 2015; 3: 484-492.

[14] Schiller E, Mechanic O. Opioid overdose. Stat Pearls. [Internet]. 2019 [cited 2019 Apr 23]; Available from: https://www.ncbi.nlm.nih.gov/books/NBK470415/

[15] Solberg C, Brown R, Du Vivier D, Meyer J, Ramachandran B. The US opioid crisis: Current federal and state legal issues. Anasthesia \& Analgesia. 2017 Nov; 125(5): 1675-1681.

[16] Weisberg D, Becker W, Fiellin D, Stannard C. Prescription opioid misuse in the United States and the United Kingdom: Cautionary lessons. International Journal of Drug Policy. 2014 Nov; 25(6): 1124-1130. 
Table 1.

CDC guidelines for prescribing opioids for chronic pain [2].

When to initiate or continue opioids:

1. Non-pharmacologic therapy and non-opioid pharmacologic therapy are preferred treatment for chronic pain. If used, opioids should be given in combination with non-pharmacologic therapy and non-opioid pharmacologic therapy when appropriate.

2. Realistic treatment goals should be established prior to initiating therapy.

3. Before starting and periodically during treatment, clinicians should discuss risks and benefits with the patient.

Opioid selection, dosing and duration:

1. Immediate release opioids should be used instead of long acting opioids.

2. The lowest effective dose should be prescribed.

3. When treating acute pain, the lowest effective dose of immediate release opioids should be used for a limited duration; typically, 3 days or less, rarely more than 7 days.

4. Clinicians should reevaluate benefits and harms with the patient 1-4 weeks after starting chronic opioid therapy and re-assess risk-benefit of medication. Assessing risk and addressing harms:

1 Evaluate risk factors for adverse events before initiation and during treatment. Consider naloxone for higher risk patients.

2. Review prescription drug monitor program (PDMP) database before initiating and during treatment.

3. Urine drug testing should be used before initiating and periodically during treatment.

4. Avoid prescribing opioids and benzodiazepines together.

5. Offer treatment or refer patients to treatment if opioid use disorders is expected 